\title{
BMJ Open Compliance with a time-out procedure intended to prevent wrong surgery in hospitals: results of a national patient safety programme in the Netherlands
}

\author{
Steffie M van Schoten, ${ }^{1}$ Veerle Kop, ${ }^{1}$ Carolien de Blok, ${ }^{1,2}$ Peter Spreeuwenberg, ${ }^{1}$ \\ Peter P Groenewegen, ${ }^{1,3}$ Cordula Wagner ${ }^{1,4}$
}

To cite: van Schoten SM, Kop V, de Blok C, et al. Compliance with a time-out procedure intended to prevent wrong surgery in hospitals: results of a national patient safety programme in the Netherlands. BMJ Open 2014:4:e05075

doi:10.1136/bmjopen-2014005075

- Prepublication history for this paper is available. To view please visit the journal (http://dx.doi.org/10.1136/ bmjopen-2014-005075).

Received 17 February 2014 Revised 23 May 2014 Accepted 2 June 2014

CrossMark

For numbered affiliations see end of article.

Correspondence to Steffie M van Schoten; s.vanschoten@nivel.nl

\section{ABSTRACT}

Objective: To prevent wrong surgery, the WHO 'Safe Surgery Checklist' was introduced in 2008. The checklist comprises a time-out procedure (TOP): the final step before the start of the surgical procedure where the patient, surgical procedure and side/site are reviewed by the surgical team. The aim of this study is to evaluate the extent to which hospitals carry out the TOP before anaesthesia in the operating room, whether compliance has changed over time, and to determine factors that are associated with compliance.

Design: Evaluation study involving observations.

Setting: Operating rooms of 2 academic, 4 teaching and 12 general Dutch hospitals.

Participants: A random selection was made from all adult patients scheduled for elective surgery on the day of the observation, preferably involving different surgeons and different procedures.

Results: Mean compliance with the TOP was $71.3 \%$. Large differences between hospitals were observed. No linear trend was found in compliance during the study period. Compliance at general and teaching hospitals was higher than at academic hospitals. Compliance decreased with the age of the patient, general surgery showed lower compliance in comparison with other specialties and compliance was higher when the team was focused on the TOP.

Conclusions: Large differences in compliance with the TOP were observed between participating hospitals which can be attributed at least in part to the type of hospital, surgical specialty and patient characteristics. Hospitals do not comply consistently with national guidelines to prevent wrong surgery and further implementation as well as further research into noncompliance is needed.

\section{INTRODUCTION}

Ideally, hospitals should be safe environments for their patients. However, making errors is inherent in all humans. ${ }^{1}$ The report 'To Err is Human' showed that errors cause 44 000-98 000 deaths and over one million

\section{Strengths and limitations of this study}

Structured observations of compliance with time-out procedure (TOP) and factors that are associated with compliance at operating rooms (ORs).

- The presence of the observer might have influenced the behaviour of the $\mathrm{OR}$ staff.

- A potential selection bias in the surgical procedures on the observation days may have occurred. No outcome data are available.

injuries each year in American hospitals. ${ }^{1}$ As a result, patient safety became a major topic on the healthcare agenda. ${ }^{2-4}$ Patient safety covers the prevention of errors and adverse events associated with healthcare that affect patients. ${ }^{5}$ An adverse event is unintentional harm caused by healthcare management rather than by the patient's underlying disease that results in a prolonged hospital stay, temporary or permanent disability or death. ${ }^{6}$ In 2004, adverse events occurred in approximately $5.7 \%$ of hospital admissions in the Netherlands: approximately $2.3 \%$ of the adverse events were potentially preventable. ${ }^{6}$ More than $54 \%$ of the unintentional adverse events were associated with the surgical procedure, of which $34 \%$ were reviewed as being preventable. $^{6}$ It is therefore important to ensure and improve patient safety during surgery.

Patient safety in surgery has several aspects. One of these aspects is wrong surgery, which can be classified into three groups: surgery at the wrong site, surgery on the wrong patient and carrying out the wrong procedure. ${ }^{7}$ Wrong site surgery occurs whenever a planned surgical procedure is performed at or on the wrong place, part and side or site. Wrong patient surgery refers to a procedure 
performed on the wrong patient. Wrong procedure surgery refers to a different procedure being performed than the one planned for the patient. The Joint Commission on Accreditation of Healthcare Organizations (JCAHO) sentinel event database ranked wrong site surgery as the second most frequently reported adverse event between 1995 and $2005 .{ }^{8}$ In the USA, for instance, the estimated rate of wrong site surgery ranges from 0.09 to $4.5 / 10000$ operations. ${ }^{3}$ 8-13

To prevent wrong surgery, the JCAHO guideline 'Universal protocol for Preventing Wrong Site, Wrong Procedure, Wrong Person Surgery' was adopted in 2003 by the Joint Commission in the USA. ${ }^{14}$ Consequently, the WHO introduced a checklist in 2008 for worldwide use, called the 'Safe Surgery Checklist'. In 2009, the WHO concluded that the use of a checklist in the operating room (OR) is associated with a significant decrease in postoperative complication (30\%) and mortality rates $(50 \%) .{ }^{15}$ Based on these results, the WHO estimated that implementing the checklist could save 500000 lives every year worldwide. ${ }^{15}$ Other studies provided evidence supporting the use of surgical checklists as well. ${ }^{16-19}$ In the Netherlands, the SURgical PAtient Safety System (SURPASS) was developed with the same intention. It is based on safety checks used in the aviation industry to reduce human error. ${ }^{20}$ Research on the external validation of the SURPASS shows a reduction in unintentional harm. ${ }^{21-23}$

Each of the checklists aforementioned comprises a time-out procedure (TOP). Errors can be avoided by including a preoperative discussion just before the start of the surgical procedure. This takes place during a time-out involving a review of the names and roles of all team members, characteristics of the patient, the operation plan, familiarity with the procedure, the presence of the correct materials/equipment and potential issues for the patient. ${ }^{24}{ }^{25}$ Although evidence is scarce, it is likely that these TOPs reduce uncertainties in the OR among the surgical team and reduce the risk of wrong surgery. The TOP is the final step before the start of the surgical procedure and is therefore crucial in preventing wrong surgery. A TOP is carried out just before anaesthesia, ${ }^{26}$ and consists of three checks (the patient, the procedure and the side/site), all of equal importance in preventing wrong surgery.

The aim of this study is to evaluate the extent to which hospitals carry out the TOP before anaesthesia in the OR, whether compliance has changed over time, and to determine factors that are associated with the TOP compliance. Insights into compliance with the TOP and the factors associated with compliance are important because they have the potential to improve the TOP and reduce adverse events in surgical processes throughout the world. This study was carried out in the Netherlands and was part of a larger evaluation study of the Dutch Hospital Patient Safety Program (hereinafter 'Safety Program') that was carried out during the final year of the programme (box 1 ).

\section{Box 1 The Dutch Hospital Patient Safety Program.}

The Dutch Hospital Patient Safety Program (Safety Program) was set up in 2008 to reduce preventable unintentional adverse events in Dutch hospitals by $50 \%$ by the end of $2012 .^{26}$ The Safety Program consisted of 10 patient safety themes and clinical guidelines were developed for each theme. Hospitals were given 5 years to implement these guidelines. One of the themes was prevention of wrong surgery. There are several risk factors for wrong surgery, for example, insufficient compliance, inadequate identification and verification and bad preoperative planning. ${ }^{27} 28$ The Safety Program therefore instructed the participating Dutch hospitals to implement several steps to decrease wrong surgery, based on the SURPASS checklist. One of the steps is identification and verification by means of a TOP consisting of checks on the correct patient, correct side, and correct intervention. ${ }^{29}$

On the basis of the goals of the Safety Program, it was expected that the compliance with the TOP would increase over time and would become more visible during the final year of the programme when hospitals approached the public deadline at the end of 2012.

The research questions are:

1. To what extent do Dutch hospitals comply with the TOP before anaesthesia in the OR?

2. How has the compliance with the TOP changed during the final year of the Safety Program?

3. What factors are associated with compliance with the TOP?

\section{METHODS}

\section{Study design}

This study was part of a larger evaluation study of the Safety Program that was carried out between November 2011 and December 2012 in 18 Dutch hospitals (about $20 \%$ of all Dutch hospitals). Hospitals were randomly selected using a stratified sample based on geographical regions and hospital type. Two academic hospitals, four teaching hospitals and 12 general hospitals were included in this study. All hospitals consented to the study and were informed about further practical issues. Twelve observers participated in this study. Inter-observer variability was not measured, but limited by training of observers prior to the start of the observations. Moreover, regular feedback meetings were held where observers exchanged experiences and discussed how to deal with certain situations and observations at the OR. A random selection was made from all adult patients scheduled for elective surgery on the day of the observation. This selection was made by the observers who were instructed to attend as many different surgeries as possible while ensuring they were present in the OR before the start of each surgery, which was essential in order to be able to observe the TOP procedure. The goal was to have 10 observation days per hospital at intervals of 4-6 weeks, and to observe 6-10 surgical procedures per day, preferably involving different surgeons 
and different procedures. One observer per surgical procedure evaluated whether the TOP was carried out before anaesthesia, using a standardised recording form that covered the various aspects of doing the TOP: checking the patient, procedure, and side/site, attention of the team (focus), completeness of the team, interruptions, and several background variables such as the type of surgical procedure, the patient's age and sex. The OR team was not aware of the exact subject matter of the observation; the observer was instructed to introduce the study in abstract terms, referring to it as a study about the surgical process in general.

\section{TOP compliance}

The outcome measure was whether the TOP was done correctly and was dichotomous (yes/no). This variable was used to examine mean TOP compliance and the changes in compliance during the study period. A correct TOP consists of three checks: patient, procedure and side/site. Since all three checks are equally important for preventing wrong surgery, the TOP was only deemed correct when all three checks were performed. Furthermore, during a TOP the entire OR team gathers around the patient and the surgeon asks the patient his/her name, the type of procedure and the side/site of the procedure.

Four independent variables were included so that any association with compliance could be determined. The type of hospital was categorised into academic, teaching, and general. In the Netherlands, teaching hospitals provide specialised medical care and are committed to training and education. The level of care can be characterised as complex and lies between that of general hospitals and academic centres. Hospital size was operationalised as the number of beds in the hospital (a continuous variable). Surgical specialty was added as a categorical variable with general surgery as the reference category. Focus (yes/no) was included to measure the degree to which the OR team was paying full attention to the TOP and was not performing any other activities during the TOP. In addition, the patient characteristics 'age' and 'sex' were included as covariates. Completeness of the team (yes/no) was added as an explorative analysis. The complete team in this study was seen as the group of persons that performed the surgery on the patient. To be able to perform a TOP correctly, the complete team was present during the TOP. When this was not the case, meaning that one or more persons joint the team after the TOP had been completed, team completeness was scores as 'no'.

\section{Statistical analyses}

Descriptive analyses were performed to obtain a picture of the study population, mean TOP compliance, changes in compliance over time, mean compliance for the different hospital types, mean compliance for the different surgical specialties, and the focus and completeness of the team during the TOP.
A multilevel logistic regression analysis with two levels was used to determine whether TOP compliance changed between the 10 measuring moments. Multilevel analysis was chosen to correct for the fact that the surgical procedures are not independent from each other, but clustered within hospitals. Time was modelled by adding 10 indicator variables for the measurement moments (removing the intercept from the model); trends were tested using polynomial contrasts (to the fourth order) to study changes over time. Variance and intraclass correlations (ICCs) were calculated to assess the clustering of TOP compliance at the hospital and surgical procedure level. An ICC of $20 \%$ was seen as moderate. ${ }^{30}$ The changes over time were also analysed for the different hospital types to determine the relationship between hospital type and the changes in TOP compliance. Separate logistic multilevel analyses were performed for each independent variable to analyse the effects of the independent variables 'hospital size' and 'surgical specialty'; this was necessary because not enough units at the highest level (hospitals) were available to have more than one independent variable in a model. ${ }^{30}$ There were not enough units at the highest level (hospitals) to model the effect of hospital type on the TOP score in the pooled analyses. Age and sex of the patient were added as covariates in all analyses. All descriptive analyses were performed using SPSS version PASW Statistics V.18. The multilevel analyses were performed using MlwiN V.2.24 (using PQL, second order, unconstrained level 1 variance, and options).

\section{RESULTS}

\section{Descriptive analyses}

A total of 1281 surgical procedures were observed at the participating hospitals. After patients younger than 18 were excluded, 1232 observations remained for analysis. Ages ranged from 18 to 96 . The gender distribution was $41.4 \%$ male, $53.8 \%$ female, and $4.8 \%$ not registered. The range in types of surgical procedures was broad; observers had been instructed to observe different procedures and observed surgical procedures of in total 13 different specialties. Mean compliance with the TOP during the total study period was $71.3 \%$. Descriptive analyses showed that TOP compliance did not improve during the study period. There was a large spread between hospitals: one of the hospitals never performed the TOP correctly and two had mean compliance rates higher than $90 \%$. A low mean TOP compliance (48\%) was found at the ninth measuring moment for all the participating hospitals. The academic hospitals had a mean compliance rate of $42.1 \%$, teaching hospitals $76.2 \%$ and general hospitals $73.9 \%$. Differences between specialties were shown to exist: trauma, gastroenterology and hepatology and ear, nose and throat medicine (ENT) had the highest compliance rates. Anaesthesiology, cardiothoracic surgery and cosmetic surgery had the lowest compliance rates. In $44 \%$ of the 
observations the team was not focused on the TOP and in $56 \%$, the team was incomplete.

\section{Multilevel regression analyses}

In the first multilevel regression analysis, the changes in TOP compliance were tested. The effect was statistically significant for the fourth-order polynomial $(p<0.01)$, meaning that TOP compliance was not linear but fluctuated over time and no clear trend was observed. Furthermore, there were large differences between the measuring moments and between individual hospitals (see figure 1). The multilevel analysis shows that $44 \%$ $(\mathrm{ICC}=44.01)$ of the total variance in TOP compliance can be attributed to the differences between the individual hospitals. Adding hospital type to the analysis caused the ICC to drop to 40.11 (40\%; see table 1$)$.

When correcting for age and sex of the patient, the ICC dropped to $26 \% \quad(\mathrm{ICC}=26.58)$. The relationship between the age of the patient and the TOP was found to be significant $(p<0.05)$. This relationship was tested and found to be linear. Based on the results described above, there was no rationale to correct for time (measurement moments) in further analyses. Observations from the different measurement moments were pooled in the remaining analyses.

Separate analyses were performed for the independent variables 'hospital size', 'surgical specialty', and 'focus'. No statistically significant relationship was found between hospital size and TOP compliance (data not shown in tables). A positive relationship was found between patients undergoing ENT surgery and the TOP (reference=general surgery; $\mathrm{p}<0.01$ ). Another positive relationship was found between patients undergoing ophthalmic surgery and the TOP (reference=general surgery; $\mathrm{p}<0.05$; see table 2 ). This indicates that TOP compliance is significantly higher in patients undergoing ENT surgery or ophthalmic surgery compared with patients undergoing general surgery. The relationship between the age of the patient and TOP compliance was found to be significant $(\mathrm{p}<0.05)$ in all analyses. This indicates that TOP compliance decreases with the patient age. The TOP is performed correctly less often for older patients. An additional analysis was performed based on these results to determine which of the three individual checks of the TOP attributed most to the negative relationship between the age of the patient and TOP compliance. Table 3 shows the results of the additional analysis. The check procedure contributes most to the negative relationship between age of the patient and TOP compliance, this check is more often skipped when an older patient is involved. The relationship between the focus of the team during the TOP and the correct execution of the TOP is shown in table 4. There is a positive significant relationship between focus and TOP compliance, which indicates that the TOP is more often correctly executed when the entire team is focused on the TOP and not performing any other activities at the same time.

\section{DISCUSSION}

The objective of this study was to investigate the compliance at Dutch hospitals with the national guidelines of a TOP set by the Safety Program and how this changed over the final year of the programme. Furthermore, we studied variables that might be associated with compliance. This study found a mean TOP compliance of $71.3 \%$. There was no linear trend in the TOP compliance during the study period. Large differences were found between and within individual hospitals, which were partly influenced by age of the patient. The type of hospital was associated with the TOP compliance: academic hospitals had lower compliance rates than general and teaching hospitals. Given the low number of academic hospitals in this study $(\mathrm{N}=2)$, these findings cannot be generalised to academic hospitals as a whole. ENT medicine and ophthalmological surgery had higher TOP compliance than the reference group (general surgery). No statistically significant relationship between TOP compliance and hospital size was found. The TOP was correctly performed more often when the OR team was focused on it. The negative relationship
Figure 1 Trend in the time-out procedure compliance per hospital type, and overall mean $(n=1232)$.

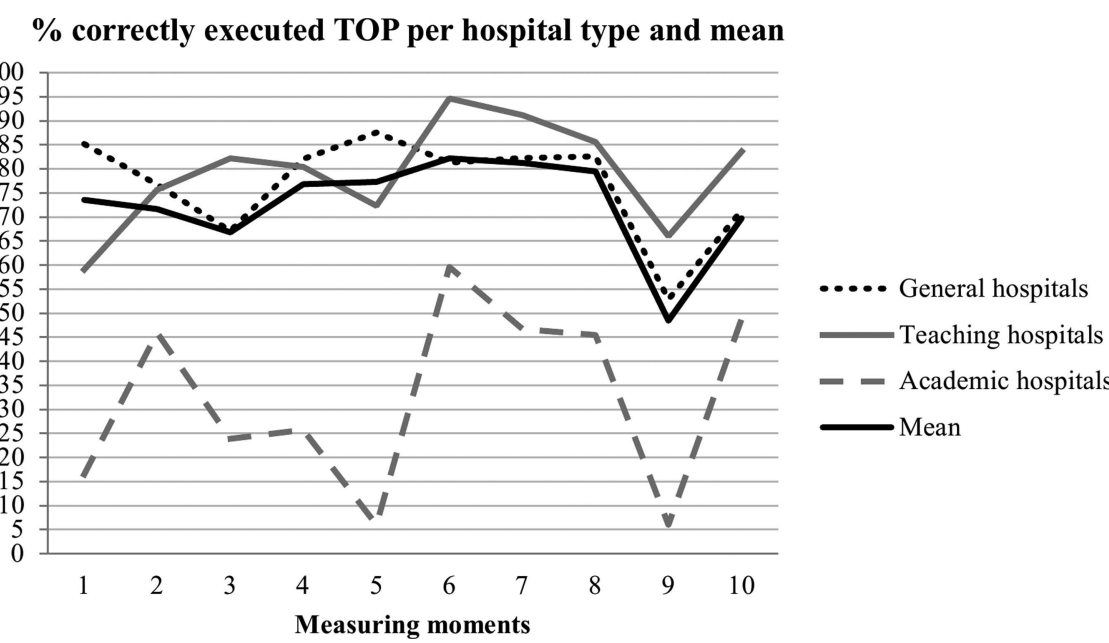


Table 1 Trend in the time-out procedure per hospital type ( $n=1232 ; 18$ hospitals)

\section{$\mathbf{N}$}

Mean \% $\quad 95 \% \mathrm{Cl}$

\section{Trend overall}

\section{Trend per hospital type}

\section{General hospitals}

N Mean \% $\quad 95 \%$ Cl

TOP (constant)

$\mathrm{MM}$

$\mathrm{MM} 2$

MM 3

MM 4

MM 5

MM 6

MM 7

MM 8

137

73.52

71.64

66.79

76.77

77.26

82.18

81.20

79.41

$\begin{array}{llll}53.20 & \text { to } 87.14 \quad 85 & 85.18\end{array}$

$\begin{array}{llll}51.26 & \text { to } 85.85 & 91 & 76.73\end{array}$

45.78 to $82.73 \quad 87 \quad 67.09$

57.01 to $89.17 \quad 75 \quad 82.01$

57.99 to $89.32 \quad 85 \quad 87.56$

64.73 to $92.05 \quad 85 \quad 81.27$

62.89 to $91.67 \quad 78 \quad 82.23$

$\begin{array}{llll}60.46 & \text { to } 90.68 \quad 82 & 82.59\end{array}$
MM 9

MM 10

48.44

28.65 to $68.74 \quad 89 \quad 52.86$

69.68

129

115

Random effects

Variance components:

ICC

44.056

Hospital (level 2)

$2.591(0.916)^{*}$

48.46 to $84.88 \quad 85 \quad 71.32$

40.106

$2.203(0.798)^{\star}$
2.00 to $94.35 \quad 25 \quad 59.00$

54.48 to $90.09 \quad 33 \quad 75.65$

42.94 to $84.66 \quad 33 \quad 82.19$

60.92 to $93.02 \quad 27 \quad 80.40$

$\begin{array}{llll}70.27 & \text { to } 95.44 & 30 & 72.30\end{array}$

60.55 to $92.46 \quad 27 \quad 94.63$

61.44 to $93.07 \quad 26 \quad 91.13$

62.33 to $93.16 \quad 22 \quad 85.56$

29.81 to $74.75 \quad 25 \quad 66.00$

47.57 to $87.20 \quad 21 \quad 83.56$
21.26 to $88.46 \quad 11 \quad 16.48$

36.60 to $94.36 \quad 13 \quad 45.91$

44.85 to $96.32 \quad 14 \quad 23.86$

41.08 to $96.02 \quad 16 \quad 25.73$

$\begin{array}{llll}32.65 \text { to } 93.36 & 10 & 5.97\end{array}$

65.68 to $99.39 \quad 15 \quad 59.61$

57.87 to $98.72 \quad 10 \quad 46.67$

$\begin{array}{llll}46.93 & \text { to } 97.54 \quad 8 & 45.42\end{array}$

26.36 to $91.33 \quad 15 \quad 6.00$

43.24 to $97.13 \quad 9 \quad 48.84$
0.91 to 80.91

2.90 to 96.02

1.55 to 86.19

1.76 to 87.03

0.29 to 57.90

5.32 to 97.48

2.40 to 96.89

1.80 to 97.43

0.40 to 50.29

2.39 to 97.38

Surgical procedure (level 1) $0.988(0.040)$

$0.984(0.040)$

$\mathrm{p}<0.05$

ICC, intraclass correlation coefficient; MM, measurement moment; TOP, time-out procedure. 
Table 2 Relationship between surgical specialties ( $n=1130 ; 18$ hospitals) and compliance with the time-out procedure

\begin{tabular}{lll}
\hline & Model $\mathbf{0}$ (time-out procedure+age+sex) & Model 1 (model 0+specialties) \\
\hline Fixed effects & Estimate (SE) & Estimate (SE) \\
Time out procedure (constant) & $1.173(0.268)$ & $1.196(0.269)$ \\
Specialties-general surgery & - & Reference \\
Specialties-gynecology & - & $0.050(0.264)$ \\
Specialties-ENT & - & $0.905(0.316)^{*}$ \\
Specialties-ophthalmology & - & $0.616(0.302)^{*}$ \\
Specialties-orthopedic surgery & - & $0.163(0.241)$ \\
Specialties-urology & - & $0.084(0.287)$ \\
Specialties-other & - & $0.046(0.279)$ \\
Patient age & $-0.011(0.004)^{*}$ & $-0.011(0.004)^{*}$ \\
Patient sex & $0.064(0.153)$ & $0.074(0.155)$ \\
Random effects & & 25.499 \\
Variance components: & & $1.126(0.426)^{*}$ \\
$\quad$ Intraclass correlation & 25.331 & $1.006(0.043)$ \\
Hospital (level 2) & $1.116(0.422)^{*}$ & \\
Surgical procedure (level 1) & $0.996(0.042)$ &
\end{tabular}

between age of the patient and the TOP indicates that higher patient age is associated with lower TOP compliance. Of all the observed TOPs, $44 \%$ were performed without the focus of the entire team, and the team was not complete in $56 \%$ of the TOPs.

A wide range in compliance rates for surgical checklists can be found in previous studies, ranging from $12 \%$ to $99 \%$ with a mean of $75 \% .^{31-33}$ The compliance rate $(71.3 \%)$ found in our study is slightly lower than the mean rate found in other studies.

We found a difference in TOP compliance between the different types of hospitals. The general and teaching hospitals hardly differed from each other, which is interesting because a previous study ${ }^{34}$ found teaching hospitals to be better at implementing checklists than general hospitals. According to the organisational learning theory, the availability of knowledge in an organisation contributes to the adoption of innovations. ${ }^{34} 35$
Teaching hospitals are learning environments, aimed at spreading and developing knowledge; better compliance can therefore be expected in teaching hospitals. We found that academic hospitals showed lower TOP compliance.

The literature is inconsistent about the influence of hospital size on the use of checklists. Some argue that larger hospitals are better developed and use standardised processes, which increases the quality of the hospital more often, ${ }^{36-38}$ whereas others conclude that smaller hospitals implement checklists better. $^{39}$ We found no relationship between TOP compliance and hospital size. The high ICC rates found in this study suggest that the differences between individual hospitals are high, and differences in compliance cannot be explained by general hospital characteristics such as hospital size. The differences between individual hospitals need to be examined in further research, but possible

Table 3 Age effects for the three different checks in the time-out procedure: checking the patient $(n=1074)$, the procedure $(\mathrm{n}=1074)$, and the side/site $(\mathrm{n}=1074)$

\begin{tabular}{llll} 
& $\begin{array}{l}\text { Model 0 (check } \\
\text { patient+age+sex) }\end{array}$ & $\begin{array}{l}\text { Model 1 (check } \\
\text { procedure+age+sex) }\end{array}$ & $\begin{array}{l}\text { Model 2 (check } \\
\text { side/site+age+sex) }\end{array}$ \\
\hline Fixed effects & Estimate (SE) & Estimate (SE) & Estimate (SE) \\
Check patient (constant) & $3.499(0.334)$ & - & - \\
Check procedure (constant) & - & $2.276(0.282)$ & - \\
Check side/site (constant) & - & - & $2.739(0.204)$ \\
Patient's age & $0.008(0.008)$ & $-0.021(0.006)^{*}$ & $0.012(0.007)^{*}$ \\
Patient's sex & $-0.185(0.288)$ & $0.124(0.198)$ & $0.160(0.246)$ \\
Random effects & & & \\
Variance components: & & 24.990 & 10.854 \\
$\quad$ Intraclass correlation & 27.172 & $1.096(0.464)^{*}$ & $0.401(0.236)^{*}$ \\
$\quad$ Hospital (level 2) & $1.228(0.623)^{*}$ & $0.922(0.040)$ & $0.950(0.041)$ \\
$\quad$ Surgical procedure (level 1) & $0.834(0.036)$ & &
\end{tabular}


Table 4 Relationship between focus ( $n=1074 ; 18$ hospitals) during the time-out procedure and compliance with the time-out procedure

\begin{tabular}{lll}
\hline & Model 0 (time-out procedure+age+sex) & Model 1 (model 0+focus) \\
\hline Fixed effects & Estimate (SE) & Estimate (SE) \\
Time-out procedure (constant) & $1.540(0.163)$ & $1.471(0.156)$ \\
Focus & - & $0.567(0.171)^{\star}$ \\
Patient's age & $-0.006(0.005)$ & $-0.005(0.005)$ \\
Patient's sex & $-0.012(0.162)$ & $-0.016(0.163)$ \\
Random effects & & 7.991 \\
Variance components: & & $0.286(0.140)^{\star}$ \\
$\quad$ Intraclass correlation & 8.971 & $0.966(0.042)$ \\
$\quad$ Hospital (level 2) & $0.324(0.154)^{*}$ &
\end{tabular}

explanations might be found in different organisational structures, the creation of awareness among healthcare staff and differences in speaking-up cultures between hospitals.

The relationship found between surgical specialties and the TOP is different from the results of previous studies. One study showed a difference between surgeons and anesthesiologists ${ }^{40}$ and another study showed no difference between surgical specialties at all. ${ }^{41}$ The TOP is a standardised procedure, and the way in which it should be carried out does not depend on the surgical specialty performing the procedure or the patient characteristics. Compliance with the TOP varied between different specialties and was lowest among general surgery teams. One explanation for these differences could be that not all medical disciplines and their scientific communities have placed the same amount of weight on a thorough implementation of the Safety Program. If so, this could have had an influence on the sense of urgency experienced by different specialties to comply with TOP in their daily functioning. Further research that includes specialty-specific factors is needed to verify and deepen our findings. The negative relationship between TOP compliance and the age of the patient was an unexpected result, since the TOP should be executed in the same way for all patients. In particular, the exact surgical procedure that would be carried out was less often verified with elderly patients. Explanations might be found in factors inherently associated with the elderly patient themselves. For example, elderly patients might be less able to verbally express themselves to healthcare staff. On the other hand, explanations might be found in factors that are associated with the medical procedure itself. For example, the level of standardisation of procedures that are commonly performed in the elderly population (such as hip-replacement surgery or cataract surgery) is relatively high and it is unclear what effect this has on compliance with TOP. Elderly people are a vulnerable group with a higher risk of complications after surgery, therefore further in-depth research is important to explain the differences in compliance for different age groups.
Completeness and focus are important factors in the TOP and performing it when team members are busy with other activities creates a risk. Our study showed that focus in the team contributes to the TOP being performed correctly. However, there was poor focus on the TOP in almost half of the surgical procedures observed. Several possible causes could be underlying to poor focus during the TOP, which was observed frequently in our study. First, there could have been a lack of awareness of the importance of the TOP among healthcare staff. Regular emphasis on the importance of the TOP during team meetings or during the joint briefing at the start of a new working day could help raise awareness. Second, when surgery schedules are tight, healthcare staff might experience time pressure. In trying to keep up with the schedule and being efficient, healthcare staff might be tempted to perform multiple tasks simultaneously which in turn could negatively affect compliance with TOP.

On the basis of these results, it seems that hospitals still have a lot to gain by carrying out the TOP properly. Qualitative research methods could provide insight into the underlying reasons and incentives of why healthcare staff perform the TOP in the way they currently do. This type of research could complement and deepen the findings that were presented in the current study.

\section{Strengths and limitations}

Our study was the first to evaluate TOP compliance over time through observations in the OR and look into the factors associated with compliance. Our dependent variable was a process indicator, because the incidence of wrong surgery is too low to be observed with our study design. Based on the literature, it seems fair to assume that higher TOP compliance can contribute to a decrease in the incidence of wrong surgery, ${ }^{15}$ although this study gives no information about the actual number of wrong surgeries and TOP compliance might not be the only factor in the reduction of wrong surgery.

This study has several limitations. First, the presence of the observer might have influenced the behaviour of the OR staff and indirectly our dependent variable TOP. 
However, the design of our study aimed to prevent this potential observer bias, because the precise goal of the observations was not known to the OR team. Second, a potential selection bias can be found in the selection of surgical procedures on the observation days. Surgical procedures were selected based on practical considerations: the day of the week, the duration of the procedure and the OR schedule. The relationships found between different specialisms might be partially overestimated, because the same surgical teams were sometimes observed on the same day or on different observation days. However, the overall goal was to observe as may different surgical procedures with different teams as possible, in order to limit potential selection bias. Third, there is no information available about the changes in compliance during the first period of the Safety Program, and hospitals may have made progress during this period.

\section{CONCLUSIONS}

The mean TOP compliance was $71.3 \%$ during the final year of the Safety Program and no improvement in compliance over time was found. Large differences were found between hospitals, and these differences were influenced by age of the patient. Compliance was influenced by several factors: hospital type, surgical specialty, age of the patient and focus of the team during the TOP. Furthermore, in almost half the TOPs, the team was not focused on the TOP or the team was incomplete. Despite the fact that almost three quarter of operations are preceded by a TOP, hospitals need to make an effort to improve TOP compliance and the way in which the TOP is carried out in order to prevent wrong surgery from happening in the future.

\section{Author affiliations}

${ }^{1}$ NIVEL-Netherlands Institute for Health Services Research, Utrecht, The Netherlands

${ }^{2}$ Faculty Economics and Business, Department Operations, University of Groningen, Groningen, The Netherlands

${ }^{3}$ Departments of Sociology and Human Geography, Utrecht University, Utrecht, The Netherlands

${ }^{4}$ Department of Public and Occupational Health, EMGO Institute for Health and Care Research, Vrije Universiteit Medical Center (VUmc), Amsterdam, The Netherlands

Acknowledgements The authors thank the participating hospitals, their contact persons and employees who participated in our study.

Contributors SMvS and VK did the statistical analyses, interpreted the results of the analyses and drafted the manuscript. SMvS took part in the data collection. CdB was involved in the design of the study, organised the data collection and critically revised the manuscript. PS did the statistical analyses and reviewed the parts of the manuscript that involved the statistical analyses. PPG was involved in the design of the study, advised on the statistical analyses and critically revised the manuscript. CW was involved in the design of the study and critically revised the manuscript. All the authors meet the criteria for authorship, were involved in revising and approving the final manuscript and accept responsibility for the data presented.

Funding This research was funded by the Dutch Hospital Safety Program and he Ministry of Education, Culture and Science in the Netherlands.
Competing interests None.

Ethics approval The study protocol was granted approval by the VU University Medical Center ethical review board in Amsterdam.

Provenance and peer review Not commissioned; externally peer reviewed.

Data sharing statement No additional data are available.

Open Access This is an Open Access article distributed in accordance with the Creative Commons Attribution Non Commercial (CC BY-NC 3.0) license, which permits others to distribute, remix, adapt, build upon this work noncommercially, and license their derivative works on different terms, provided the original work is properly cited and the use is non-commercial. See: http:// creativecommons.org/licenses/by-nc/3.0/

\section{REFERENCES}

1. Kohn LT, Corrigan J, Donaldson MS. To err is human: building a safer health system. 6th edn. Joseph Henry Press, 2000.

2. See LC, Chang YH, Chuang KL, et al. Animation program used to encourage patients or family members to take an active role for eliminating wrong-site, wrong-person, wrong-procedure surgeries: preliminary evaluation. Int J Surg 2011;9:241-7.

3. Seiden SC, Barach P. Wrong-side/wrong-site, wrong-procedure, and wrong-patient adverse events: are they preventable? Arch Surg 2006;141:931.

4. Zhan C, Kelley E, Yang HP, et al. Assessing patient safety in the United States: challenges and opportunities. Med Care 2005;43:42-7.

5. WHO. Patient Safety. 2013. http://www.who.int

6. Zegers M, De Bruijne MC, Wagner C, et al. Adverse events and potentially preventable deaths in Dutch hospitals: results of a retrospective patient record review study. Qual Saf Health Care 2009;18:297-302.

7. Gibbs VC. Thinking in three's: changing surgical patient safety practices in the complex modern operating room. World $\mathrm{J}$ Gastroenterol 2012;18:6712.

8. DeVine J, Chutkan N, Norvell DC, et al. Avoiding wrong site surgery: a systematic review. Spine 2010;35:S28-36.

9. Ammerman JM, Ammerman MD, Dambrosia J, et al. A prospective evaluation of the role for intraoperative x-ray in lumbar discectomy. Predictors of incorrect level exposure. Surg Neurol 2006;66:470-3.

10. Kwaan MR, Studdert DM, Zinner MJ, et al. Incidence, patterns, and prevention of wrong-site surgery. Arch Surg 2006;141:353.

11. Jhawar BS, Mitsis D, Duggal N. Wrong-sided and wrong-level neurosurgery: a national survey. J Neurosurg Spine 2008;9:109.

12. Mody MG, Nourbakhsh A, Stahl DL, et al. The prevalence of wrong level surgery among spine surgeons. Spine 2008;33:194.

13. Clarke JR, Johnston J, Finley ED. Getting surgery right. Ann Surg 2007;246:395.

14. Angle JF, Nemcek AA Jr, Cohen AM, et al. Quality improvement guidelines for preventing wrong site, wrong procedure, and wrong person errors: application of the joint commission "universal protocol for preventing wrong site, wrong procedure, wrong person surgery" to the practice of interventional radiology. $J$ Vasc Interv Radiol 2009;20(7 Suppl):S256-62.

15. World Health Organization. New scientific evidence supports WHO findings: a surgical safety checklist could save hundreds of thousands of lives. 2011. http://www.who.int

16. Haynes AB, Weiser TG, Berry WR, et al. A surgical safety checklist to reduce morbidity and mortality in a global population. $N$ Eng $J$ Med 2009;360:491-9.

17. Mazzocco K, Petitti DB, Fong KT, et al. Surgical team behaviors and patient outcomes. AM J Surg 2009;197:678-85.

18. Gawande AA, Zinner MJ, Studdert DM, et al. Analysis of errors reported by surgeons at three teaching hospitals. Surgery 2003;133:614.

19. Lübbeke A, Hovaguimian F, Wickboldt N, et al. Effectiveness of the surgical safety checklist in a high standard care environment. Med Care 2013;51:425-9.

20. Hales BM, Pronovost PJ. The checklist: a tool for error management and performance improvement. J Crit Care 2006;21:231-5.

21. De Vries EN, Prins HA, Crolla RM, et al. Effect of a comprehensive surgical safety system on patient outcomes. $N$ Eng J Med 2010;363:1928-37.

22. De Vries EN, Eikens-Jansen MP, Hamersma AM, et al. Prevention of surgical malpractice claims by use of a surgical safety checklist. Ann Surg 2011;253:624. 
23. De Vries EN, Prins HA, Bennink MC, et al. Nature and timing of incidents intercepted by the SURPASS checklist in surgical patients. BMJ Qual Saf 2012;21:503-8.

24. DeFontes J, Surbida S. Preoperative safety briefing project. Perm $\mathrm{J}$ 2004;8:21-7.

25. Nederlandse Vereniging voor Anesthesiologie, Nederlandse Vereniging voor Heelkunde. Richtlijn: Het peroperatieve traject, 2011.

26. VMS Safety Program. Prevention of wrong surgery. 2009. http:// www.vmszorg.nl

27. Joint commission. Lessons learned: wrong site surgery. 28 August 1998. http://www.jointcommissioninternational.org

28. MediRisk. OK-project: Schadecategoriën en vangnetcriteria voor de operatieafdeling. 2007.

29. De Blok C, Koster E, Schilp J, et al. Implementatie VMS Veiligheidsprogramma. Evaluatieonderzoek in Nederlandse Ziekenhuizen. (In Dutch). Utrecht/Amsterdam: NIVEL en EMGO+ Instituut, 2013.

30. Twisk JW. Applied multilevel analysis: a practical guide. Cambridge University Press, 2006

31. Spence J, Goodwin B, Enns C, et al. Student-observed surgical safety practices across an urban regional health authority. BMJ Qual Saf 2011;20:580-6.

32. Altpeter T, Luckhardt K, Lewis JN, et al. Expanded surgical time out: a key to real-time data collection and quality improvement. J Am Coll Surg 2007;204:527-32.
33. Borchard A, Schwappach DL, Barbir A, et al. A systematic review of the effectiveness, compliance, and critical factors for implementation of safety checklists in surgery. Ann Surg 2012;256:925-33.

34. Dijkstra R, Wensing M, Thomas R, et al. The relationship between organisational characteristics and the effects of clinical guidelines on medical performance in hospitals, a meta-analysis. BMC Health Serv Res 2006:6:53.

35. Nevis EC, DiBella AJ, Gould JM. Understanding organizations as learning systems. Sloan Manage Rev 1997:36:73-85.

36. Zuiderent-Jerak T, Kool T, Rademakers J. De relatie tussen volume en kwaliteit van zorg: tijd voor een brede benadering. 2012. Utrecht, Nijmegen, Rotterdam; Consortium Onderzoek Kwaliteit van Zorg, 2012:135.

37. Mutter RL, Wong HS, Goldfarb MG. The effects of hospital competition on inpatient quality of care. Inquiry 2008;45:263-79.

38. Sari N. Do competition and managed care improve quality? Health Econ 2002;11:571-84.

39. Soria-Aledo V, Da Silva ZA, Saturno PJ, et al. Difficulties in implementing a surgical check list in operating theatres. Cir Esp 2012;90:180.

40. De Vries EN, Hollmann MW, Smorenburg SM, et al. Development and validation of the SURgical PAtient Safety System (SURPASS) checklist. Qual Saf Health Care 2009:18:121-6.

41. Blanco M, Clarke JR, Martindell D. Wrong site surgery near misses and actual occurrences. AORN J 2009;90:215-22. 